Article

\title{
metaRE R Package for Meta-Analysis of Transcriptome Data to Identify the cis-Regulatory Code behind the Transcriptional Reprogramming
}

\author{
Daria D. Novikova ${ }^{1,2}$, Pavel A. Cherenkov ${ }^{3}$, Yana G. Sizentsova ${ }^{1}$ and Victoria V. Mironova $^{1,3, *(D)}$ \\ 1 Institute of Cytology and Genetics, Lavrentyeva avenue 10, 630090 Novosibirsk, Russia; \\ da6ik777@gmail.com (D.D.N.); sizentsova.yans@gmail.com (Y.G.S.) \\ 2 Laboratory of Biochemistry, Wageningen University, Stippeneng 4, 6708WE Wageningen, The Netherlands \\ 3 Novosibirsk State University, 2 Pirogova Street, 630090 Novosibirsk, Russia; cheburechko@gmail.com \\ * Correspondence: victoria.v.mironova@gmail.com
}

Received: 12 May 2020; Accepted: 5 June 2020; Published: 9 June 2020

check for updates

\begin{abstract}
At the molecular level, response to an external factor or an internal condition causes reprogramming of temporal and spatial transcription. When an organism undergoes physiological and/or morphological changes, several signaling pathways are activated simultaneously. Examples of such complex reactions are the response to temperature changes, dehydration, various biologically active substances, and others. A significant part of the regulatory ensemble in such complex reactions remains unidentified. We developed metaRE, an R package for the systematic search for cis-regulatory elements enriched in the promoters of the genes significantly changed their transcription in a complex reaction. metaRE mines multiple expression profiling datasets generated to test the same organism's response and identifies simple and composite cis-regulatory elements systematically associated with differential expression of genes. Here, we showed metaRE performance for the identification of low-temperature-responsive cis-regulatory code in Arabidopsis thaliana and Danio rerio. MetaRE identified potential binding sites for known as well as unknown cold response regulators. A notable part of cis-elements was found in both searches discovering great conservation in low-temperature responses between plants and animals.
\end{abstract}

Keywords: meta-analysis; transcription factor; binding sites; genomics; transcriptomics; chilling stress; CBF; DREB; CAMTA1

\section{Introduction}

More than two decades have passed since the establishment of whole-genome expression profiling methods. Nowadays, thousands of transcriptomes are publicly available. Typically, several related experiments studying the same phenomenon can be found, thus, providing a rich set of material for analysis. Meta-analysis is applicable to sets of experiments testing the same hypotheses to extract robust signals and repetitive features that are impossible to derive from the individual experiments.

The typical example of meta-analysis is the definition of robust differentially expressed genes (DEGs) over many transcriptomic datasets. This approach is widely used in medical genomics to identify the gene signatures associated with a condition or disease, e.g., in [1-3]. To account for the most reliable and reproducible gene signatures, different authors applied such meta-analysis procedures as Fisher's methods, Stouffer's method, permutation, or machine-learning procedures. Recently, a ready-to-use framework GSMA has been developed to solve this task for any problem of interest [3].

Alternatively, a meta-analysis of transcriptome datasets can help to understand the cis-regulatory code behind the transcriptional response. The simplest way is to analyze the upstream regions of the 
robust DEGs for overrepresented sequences, e.g., as in [4,5]. However, the way to detect the robust gene sets might be comprehensive. He and coauthors (2016) analyzed DEGs in nine transcriptomic datasets on breast cancer: DEGs were identified by Fisher's method for $p$-values combination [6]. Subsequent enrichment analysis of motifs in promoters of DEGs was estimated by Fisher's exact test and allowed identifying transcription factors associated with breast cancer.

A better way to identify a full set of cis-elements, or a "cistrome", associated with a transcriptional response, is a meta-analysis of individual transcriptomes and not the robust DEGs. Authors of the cis-Metalysis program performed a meta-analysis of transcriptomics data on bee [7]. They revealed enrichment of transcription factors binding sites in the DEGs and their association with external factors that cause similar changes in the organism. An interesting approach has been applied to study the cistrome for iron deficiency response in Arabidopsis (Arabidopsis thaliana) roots [8]. Authors searched for the enrichment of $k$-mers in upstream regulatory regions of Fe-responsive genes taken from several experiments. They applied the machine learning algorithm, Random Forest, to identify enriched elements in different functional clusters of coexpressed genes revealed. However, on the different steps of their study, authors used separate tools and approaches aiming at a specific goal of identifying clusters of Fe-responsive genes regulated by the same pulls of cis-regulatory elements.

The methods for comprehensive meta-analysis of transcription profiles for cis-elements prediction described above have proven to be powerful in specific studies. However, they were not implemented in a ready-to-use package. Here, we developed a powerful but versatile pipeline for cistrome-wide meta-analysis, implemented as a metaRE $\mathrm{R}$ package. In this study, we show the performance of $m e t a R E$ on cold-stress-responsive and hypothermia-responsive transcriptome datasets in Arabidopsis and zebrafish.

\section{Materials and Methods}

\section{1. metaRE R Package Structure and Functionality}

metaRE $\mathrm{R}$ package implements a pipeline to search for consensus sequences enriched in the promoters of DEGs. Its logic and methodology have been described in our earlier work [9], Here, we present the $\mathrm{R}$ package for the first time. We used $\mathrm{C}++$ to speed up slow components and the $R c p p$ package to integrate the $C++$ code into $\mathrm{R}$ [10]. metaRE package performs a five-step analysis: (1) DEGs identification; (2) cis-regulatory consensus element search; (3) calculation of association between consensus presence and changes in gene expression; (4) meta-analysis over multiple datasets; (5) permutation test. The pipeline is detailed below and in Figure 1.

Software with source files, documentation, and example data files are freely available online at the repository (https://github.com/cheburechko/MetaRE).

\subsubsection{DEGs Identification}

As an input, metaRE uses transcriptome data. For users' convenience, we applied GEOquery [11], limma [12], and edgeR [13,14] packages to identify DEGs in the datasets from the GEO database [15]. metaRE function prepare GEO allows loading and adjusting the preprocessed GEO data frames. Functions processMicroarray and processRNAcounts could be used to identify DEGs in a single dataset using limma (microarray and RNA-seq, respectively), functions generate a new table for a particular experiment with user-defined expression classes. The function preprocessGeneExpressionData can perform the same analysis for multiple datasets at once, it generates the final data frame GeneClassificationMatrix, which combines information about DEGs from all experiments in the meta-analysis. Alternatively, the user can upload a data frame with already processed data on differentially expressed genes.

\subsubsection{Cis-Regulatory Consensus Elements Search}

Another input data for the metaRE package are the regulatory region sequences in fasta format. MetaRE uses the Biostrings R package [16] to upload the sequences from BioMart [17]. Next, metaRE 
annotates each sequence for the presence of a potential cis-element in the following format. Function enumerateOligomers searches for all possible $k$-mers without considering complementarity, e.g., in the case of hexamers, metaRE searches for 2080 nonredundant hexamers comprising 2016 complementary pairs and 64 palindromes instead of 4096 possible combinatorial variants. In addition to $k$-mers, it is possible to annotate systematically the regulatory regions with the information about all possible spaced repeats with the same $k$-mer as a core (enumerateRepeats), spaced bipartite elements with different $k$-mers as the cores (enumerateDyadsWithCore). It is also possible to search for a predetermined list of motifs described with 15 letters IUPAC ambiguity code (enumeratePatterns). For the enumerateRepeats and enumerateDyadsWithCore functions, it is possible to set maximum and minimum spacer length in both cases. MetaRE will search for $k$-mers' combinations with given spacer length diapason. For all the functions, the logic remains the same: reverse complement $k$-mers are considered to be the same element. Thus, the number of $k$-mers/bipartite elements/repeats/predetermined motifs in the analysis is reduced compared to the number of possible combinatorial variants.

The output of the second step of the procedure is a named list of integer vectors. Names are the consensus sequence; vectors are the indices of genes in which these sequences are present.

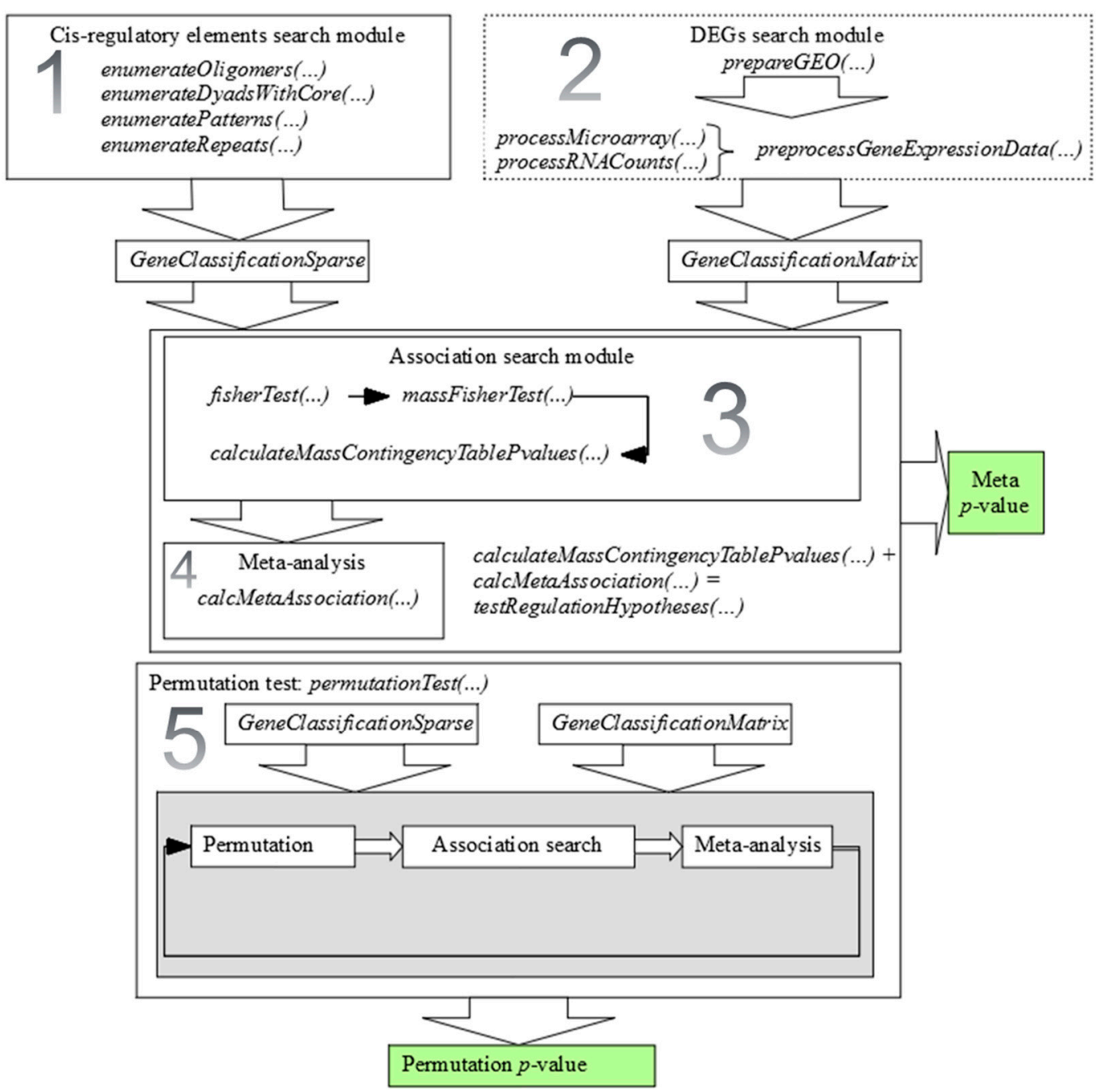

Figure 1. Scheme of metaRE modules that implement a five-step pipeline of the search for cis-elements significantly associated with differential gene expression over multiple datasets. DEGs-differentially expressed genes. Different modules are highlighted with squares; final sets of $p$-values are painted green. Described in the Methods steps are enumerated on the figure. 
2.1.3. Calculation of Association Between Cis-Regulatory Element Presence and Changes in Gene Expression

At this step, for each $k$-mer and each experiment, an association with differentially expressed genes is estimated, separately for all regulation classes. A $p$-value for the association is calculated using a $2 \times 2$ contingency table by Fisher's exact test $[9,18,19]$. The test estimates the probability of getting such an association between two variables in the contingency table. In this case, the variables are "presence/absence of the $k$-mer" and "DEG/non-DEG". In metaRE, the procedure is implemented by a function calculateMassContingencyTablePvalues. The result is a float matrix of $p$-values for the association between the $k$-mer presence and up/downregulation, where, rows correspond to the $k$-mers, columns correspond to the datasets in which cells are calculated $p$-values.

\subsubsection{Meta-Analysis}

Function calcMetaAssociation used to combine the $p$-values calculated for a particular $k$-mer over many datasets. MetaRE uses Fisher's method to calculate meta- $p$-values (Figure 1, [9]). Due to multiple testing for many $k$-mers, calcMetaAssociation also estimates an adjusted $p$-value, for which the user can choose one of the following multiple correction methods: Bonferroni, Bonferroni-Holm [20,21], Benjamini-Hochberg [22], and Benjamini-Yakuteli [23]. Users also can set the cutoff threshold for adjusted meta- $p$-value- the $k$-mers which pass the cutoff are to be tested on Step (5).

\subsubsection{Permutation Test}

Finally, metaRE applies the permutation test to the $k$-mers with significantly adjusted meta- $p$-values. MetaRE uses the foreach package (CRAN project) for parallel permutation testing. PermutationTest function shuffles the regulatory regions between the genes and recalculates meta- $p$-value for each $k$-mer in the analysis. We optimized the procedure so that every iteration-run permutationTest stores the preliminary results in "outfile" and removes the $k$-mer that will not pass the cutoff threshold. After performing $M$ permutations, the function computes the permutation $p$-value for $k$-mers left in the analysis as $p=(m+1) /(M+1)$, where $m$ is a number of recorded $p$-values not greater than the meta- $p$-value. It also computes adjusted permutation- $p$-values to consider the multiple testing (for the amount of $k$-mers predetermined on Step (4)).

In the end, the $k$-mers with an adjusted permutation- $p$-value below the cutoff threshold are considered to be significantly associated with the differential expression.

\subsection{Motifs Comparison}

To annotate predicted cis-elements, we used the TOMTOM tool from Meme Suit [24] with the reference databases DAPv1, PBM, and Cis-BP. The best match with E-value $<0.05$ was taken into the annotation.

\subsection{Datasets}

Arabidopsis and zebrafish transcriptome datasets on low positive temperature treatment were retrieved from the GEO database. 22 out of 40 datasets for Arabidopsis thaliana and 16 out of 24 datasets for Danio rerio passed the quality control for well-clustered replicas giving a sufficient number of DEGs (see Table S1). The identification of DEGs was made using the Benjamini-Hochberg method [22] to control the False Discovery Rate (FDR $<0.05$ ).

\section{Results}

\subsection{MetaRE R Package for Cistrome-Wide Association Study}

We developed a metaRE $\mathrm{R}$ package which identifies the cistrome associated with the case of study via a meta-analysis of multiple transcriptomic experiments. MetaRE pipeline includes five 
steps: (1) DEGs identification in many transcriptomic datasets, (2) search for cis-regulatory elements in upstream gene sequences, (3) assessment of the association between cis-regulatory element presence and the changes in gene expression in each transcriptomic dataset, (4) meta-analysis over multiple datasets, and (5) permutation test to study the robustness of the prediction. The first step is performed in metaRE using standard $\mathrm{R}$ packages, or the user can upload processed data. At the second step, metaRE generates the information about the presence/absence of all combinatorially possible nucleotide sequences of a particular length and structure (encoded in the 15-nucleotide IUPAC alphabet) in a set of nucleotide sequences (for instance, promoter regions, transcription factors binding regions, etc.). We considered these short nucleotide sequences as potential regulatory elements of genes' expression. Since metaRE performs a search in the promoters which are located in cis-position relative to the genes, enriched in these promoters' sequences are predicted as potential cis-acting elements. The package allows the user to identify potential cis-regulatory elements of different lengths, which could consist of one element, repeats, or bipartite elements with a variable or fixed spacer and order of elements. In the third step, metaRE assesses the association between each cis-elements and differential gene expression in each of the datasets. At the fourth step, metaRE combines the $p$-values taken from the separate datasets and highlights which of the cis-elements are systematically overrepresented. In the last step, metaRE tests the independence of obtained results from external factors by the permutation test.

The main advantage of the metaRE package is that it identifies a reliable and reproducible set of potential cis-regulatory elements associated with the transcriptional response over many independent datasets, rather than in a single gene set. The $\mathrm{R}$ package can be used for the study cases on any organism with a sequenced genome. It is possible to adjust the procedure by changing the statistical tests, thresholds, cis-elements structure, promoters' length, etc. Other nucleotide sequences could be used instead of the promoters, e.g., 3'UTRs or ChIP-Seq profiles. Thus, metaRE gives the user freedom to adjust the package to the particular study, which is essential considering the differences and quality of raw data, annotation of the genome of different species, and knowledge on the location of cis-regulatory elements.

MetaRE was tested in several independent studies on different organisms, for instance, cold-induced zebrafish transcriptomes, dioxin-induced human and mouse transcriptomes, and auxin-induced Arabidopsis transcriptomes [9]. The application of metaRE was efficient for all of the cases. Here, we discuss metaRE performance to identify cold-responsive cistrome in Arabidopsis and zebrafish.

\subsection{MetaRE for Identification of Cold-Responsive Cistrome}

To demonstrate the utility of the metaRE package, we performed analysis on cold stress-induced transcriptomes in two model objects from animal and plant fields. All the datasets so far generated with good quality for Arabidopsis thaliana and Danio rerio (Table S1, [5,25-33]) have been processed independently using metaRE. On Step (1), metaRE identified DEGs (FDR < 0.05) lists for all of 22 and 16 transcriptomic datasets. We varied the threshold for fold-change from none to 1.5 and 2 . As a result, three summary tables were generated for each organism summarizing information about the differential transcriptional response.

On Step (2), metaRE loaded Arabidopsis' and zebrafish' upstream regulatory regions [-1500; -1] of protein-coding genes from Ensemble BioMart Database (TAIR10 for Arabidopsis thaliana and GRCz11 for Danio rerio) $[17,34]$. metaRE annotated the upstream regions by the diversity of nonredundant $k$-mers. In this study, we searched for hexa-, hepta-, and octamers.

On Steps (3-5), metaRE identified all $k$-mers associated with the transcriptional cold stress response, separately for Arabidopsis and zebrafish. As the number of datasets for Arabidopsis allowed us to study time-resolved response, these cold-responsive transcriptome datasets were divided into two groups by the time of response: early response (up to six hours of cold exposure), and late response (12-24 h of cold exposure). We tried two multiple testing corrections (Bonferroni-Hochberg 
or Bonferroni) and set the stringent threshold for adjusted meta- $p$-value $<1 \times 10^{-10}$ and adjusted permutation $p$-value $\leq 1 \times 10^{-3}$.

The summary tables for identified $k$-mers (Tables S2-S4) suggest that the cistrome size provided by metaRE depends on the parameter settings. However, the most significantly enriched cis-elements remain always the same. Noteworthy, to detect any motif associated with downregulation, we had to get rid of the threshold for fold-change to identify DEGs only by FDR. Despite a more stringent multiple testing correction applied for heptamers and octamers, metaRE found more of them in this study, compared to the number of significantly overrepresented hexamers (Tables S2-S4). This was not the case in another meta-analysis performed by metaRE [9]. We can explain this fact by significant enrichment of many degenerated A/T-rich motifs in the transcriptional response to cold for both Arabidopsis and zebrafish (Figure S1; discussed below). To sum up, we recommend performing a preliminary analysis under different settings to define the most appropriate one. Below we discuss only the results obtained under the stringent Bonferroni criterium for hexamers.

\subsection{Analytics on Cold-Stress-Responsive Cistrome for Arabidopsis thaliana}

We detected 95/43 and 10/26 hexamers associated with up- and downregulation in the early/late cold stress response (Table 1). A strong bias in a cistrome diversity was detected towards the early activatory response, but apparently, it correlates with many AT-rich elements found overrepresented in the upstream regions of early cold-responsive genes (even more AT-rich motifs were found in septamers and octamers; Figure S1; Tables S2 and S3). Another trend is that cold-responsive cistrome has fewer cis-elements associated with downregulation than with upregulation. With only one exception, E-box CACGTG, hexamers were explicitly associated with either up- or downregulation.

Table 1. Summary of predicted hexamers associated with cold stress response in Arabidopsis.

\begin{tabular}{ccc}
\hline & Early Response $(<\mathbf{6} \mathbf{h})$ & Late Response $(>\mathbf{1 2} \mathbf{h})$ \\
\hline $\mathrm{Up}$ & 95 & 43 \\
Down & 10 & 26 \\
\hline \multicolumn{3}{c}{ Without A/T-rich hexamers } \\
\hline $\mathrm{Up}$ & 25 & 40 \\
Down & 10 & 26 \\
\hline
\end{tabular}

Next, we applied the TOMTOM tool [24] to annotate the predicted cis-elements associated with early and late cold response. We were able to annotate more than $65 \%$ of detected cis-elements, however, many AT-rich elements and elements related to downregulation remained unidentified (Tables S2-S3). Many of the hexamers associated with the cold stress response significantly match the binding sites of known cold response regulators from CAMTA, AP2/ERF, bHLH, MYB, and bZIP families (Figure 2A) and this fitness confirms the adequacy of metaRE pipeline.

The binding sites for C-REPEAT BINDING FACTORs (CBFs) transcription factors from AP2/ERF family (CCGACA, ACCGAC; GCCGAC, CCGACC) were expected to be found as associated with the transcriptional cold response, as CBFs are the major regulator of cold acclimation [35-38]. However, CBF binding sites were not the most abundant and significant in early response (Table S2). The most significantly enriched in early response to cold stress motifs appeared to be: (1) ACGCGT (adjusted meta- $p$-value $\left.=5.96 \times 10^{-84}\right)$, the potential binding sites for CAMTA; $(2)$ CACGTG $\left(p=1.52 \times 10^{-54}\right)$, the G-box bound by bHLH and bZIP transcription factors; (3) ACACGT $\left(p=2.3 \times 10^{-53}\right)$, the motif bound by NAC, BES, bZIP, and bHLH transcription factors; (4) ACGTGG $\left(p=2.65 \times 10^{-52}\right)$, potential binding site for bZIP and bHLH; and (5) a group of AT-rich elements $\left(3.47 \times 10^{-11}<p<2.89 \times 10^{-50}\right)$. The involvement of transcription factors bound to (1) - (4) with the cold response was known beforehand [28,39-44]. However, the fact that they are more relevant to early cold response comparing CBF binding sites is tempting, as CBF factors were recently shown to be involved in freezing not 
chilling resistance and may not be essential to survive in response to low positive temperatures [33,45]. Potential binding sites for CBFs were found the most significant for the late response to cold (Table S3).

A.
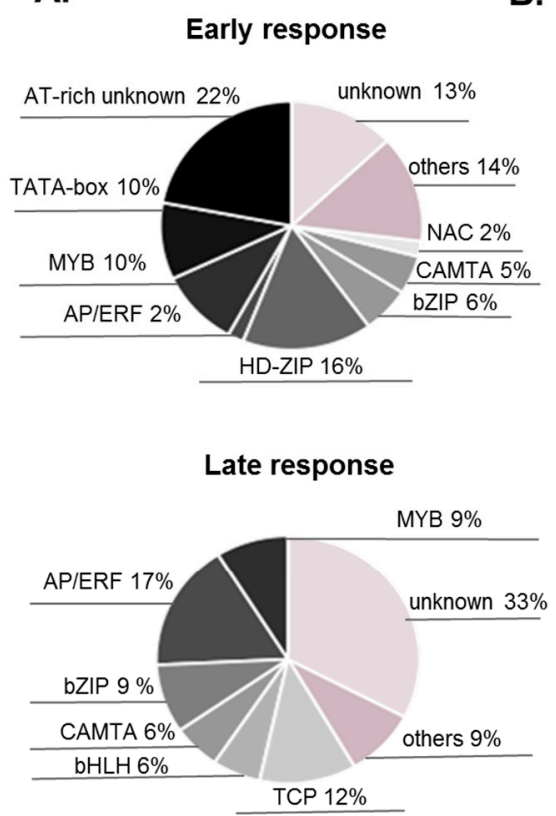

B.

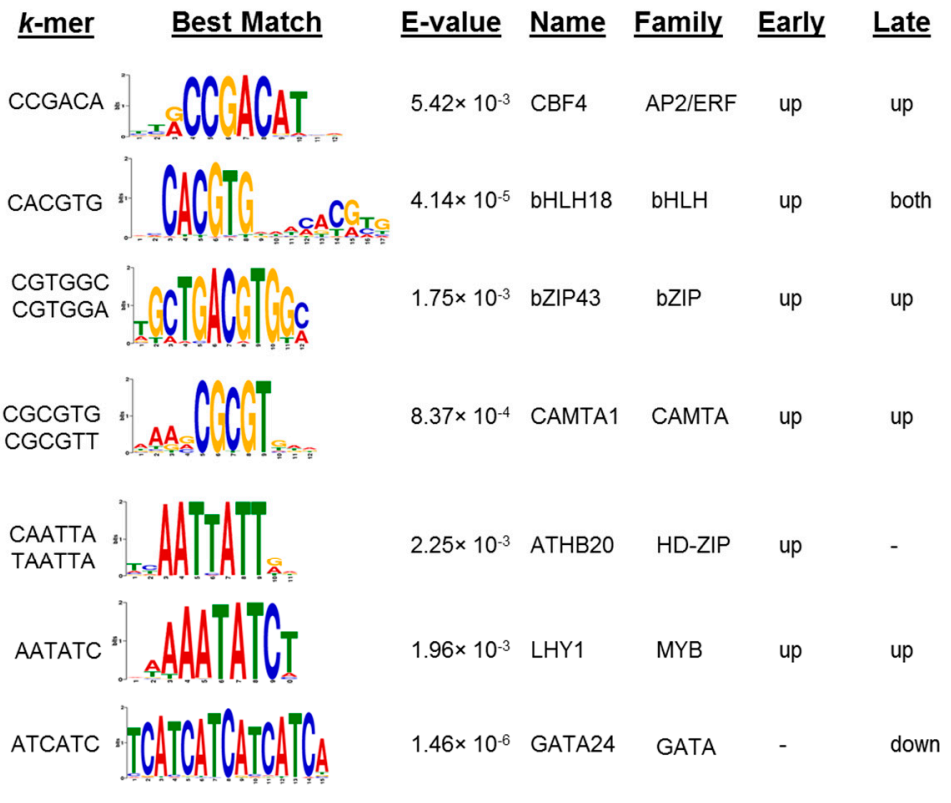

Figure 2. Cis-regulatory elements predicted with metaRE as systematically enriched in upstream regulatory regions of cold-induced genes in Arabidopsis. (A) Annotation of the hexamers to the known binding sites of Arabidopsis thaliana with the help of the TOMTOM tool [24]. Only significant best matches (E-value $<0.05$, one per hexamer) were calculated to build the round diagram. (B) Annotation details for particular hexamers associated with early, late, or both early and late responses. The best significant matches of the hexamers with the known binding sites associated with downregulation in response to cold stress.

However, most of the detected AT-rich elements remain unknown; some of these sequences significantly match (TOMTOM, E-value $<0.05)$ the known binding sites for HD-ZIP and MYB families (Table S3, Figure 2B). Although it is not clear if the detected association with HD-ZIP transcription factors is relevant, the involvement of LHY1 and CCA1 MYB transcription factors into cold stress has been discussed in several works [39,46-48]. The motifs associated with downregulation were also poorly annotated. Among the rare examples of annotated motifs associated with downregulation are GATGAT/ATCATC, the potential binding site of GATA transcription factors (Figure 2B), and a family of potential TCP-binding motifs (Table S3). These results demonstrate the perspectives of metaRE usage in the study of the cis-regulatory code behind transcriptional reprogramming in complex reactions. It allows not only predicting the diversity of involved cis-elements and respective transcription factors but also ranking them and clarifying their role in certain phases of transcriptional response.

\subsection{Analytics on Hypothermia-Related Cistrome for Danio rerio}

A similar study for zebrafish yielded 67 hexamers enriched in promoters of hypothermia-induced genes. As for predicted cold-associated elements in Arabidopsis, most of the zebrafish ones are associated with upregulation and there are many A/T-rich hexamers (Table S4). The only motif associated with both upregulation and downregulation is CGGAAG, the potential binding site for ETS transcription factor Elk1 (E-value $<2.64 \times 10^{-4}$ ). In vertebrates, the role of Elk1 transcriptional activator was widely discussed in relation to many developmental processes $[49,50]$, but not in the response to the low-temperature stress. In Danio rerio, it was only shown that Elk1 and its homologs express around the developing bone [51]. Unfortunately, cis-elements and transcription factors from Danio rerio genome are much less annotated comparing to Arabidopsis. We were not able to annotate 
overlapped hexamers (AAACGT, AACGTT, and ACGTTA), that show the greatest association with the hypothermia condition, using publicly available data. However, we assume that they compose the binding site for zebrafish' transcriptional regulator(s) that mediate the low-temperature responses.

One-third of hypothermia-related hexamers have been annotated using TOMTOM (E-value $<0.05)$. Among them: (1) two groups of AT-rich motifs resembling the binding sites for Dmrt2a (AATTTA, ATACAT, AATATA, ATAAAT, AATGTA, $2.61 \times 10^{-32}<p<8.09 \times 10^{-22}$ ) and the binding sites for Homeobox transcription factors (CATAAA, AATTAA, ATAAAA, $p<3.7 \times 10^{-11}$ ); (2) potential binding sites for bHLH transcription factors ACATAT $\left(p=2.19 \times 10^{-22}\right)$ and CACGTG $\left(p=4.4 \times 10^{-17}\right)$; (3) potential bZIPs binding sites (CGTCAC, CCGCCA, GACGTA, $p<8.74 \times 10^{-14}$ ); (4) ACCAAT, the binding site for Nfya $\left(p=5.28 \times 10^{-18}\right)$, and many others (Table S4). E-box CACGTG, A/T-reach sequences, and Nfya binding sites have been associated with the cold stress response in zebrafish earlier [33,52]. Although we have not found in the literature strong evidence for the other hypothermia-related elements to mediate low-temperature response, this might be due to the fact that this topic is largely understudied in zebrafish [33].

Unexpectedly, but a notable part of hypothermia-related motifs (27 out of 67) identified by metaRE for Danio rerio matched those identified as cold-responsive for Arabidopsis. Among them E-box CACGTG and a group of A/T-rich elements. We discuss this finding further in Section 4.2.

\section{Discussion}

\section{1. metaRE Tool for Identification of Cis-Regulatory Elements Repertoire}

The main idea behind the method implemented in the metaRE R package is that if the cis-regulatory elements are involved in a transcriptional response, then they should be overrepresented in the promoters of differentially expressed genes. This idea is not new, and there are many approaches facilitating the analysis of cis-elements overrepresentation within upstream regions of pre-compiled gene sets, e.g., in [6,53-55]. The pipeline which analyzes cis-elements overrepresentation systematically and summarizes the output taken from many independent datasets has been still required, these tasks were solved in the metaRE R package.

The novelty of the metaRE method lies in: (1) taking into account a large number of comparable transcriptome experiments, and (2) the consideration of enrichment significance for an individual cis-element. Usually, authors evaluate the enrichment of cis-elements in one or more gene lists independently; the results of enrichment between the lists are not compared [4,5]. In this case, information about differences in the degree of enrichment of the same cis-element in different datasets is leveled, which can lead to over- and underpredictions. The method underlying metaRE solves this problem.

Separate studies showed that systematic analysis of transcriptome datasets is powerful in the identification of the cistrome behind a complex reaction $[7,8,19]$. The basic assumption in these studies, as well as in the metaRE algorithm, is that only robust and significant cis-element association with transcriptional response will be detected across multiple, diverse transcriptomic datasets that test similar experimental variables. This could be considered both as an advantage and as a disadvantage of the systematic analysis. On the one hand, analysis of several datasets excludes a bias that could be caused by separate experiments (tissue sampling, treatment duration, concentration, growth conditions, quality of data, etc.). Thus, meta-analysis would detect the major cis-elements that operate under a variety of conditions. On the other hand, this approach will miss rare and condition-specific cis-elements. The latter could be solved by separate analysis of the datasets from experiments performed on different tissues, so one can have a tissue-specific cistrome. For example, in this study for cold-stress-responsive cistrome, as well as in [9] for auxin-regulated cistrome, we saw apparent differences in time-resolved results. If the number of transcriptomes allowed, these differences would be detected for tissue- and condition-specific reactions. 
Cis-elements enrichment analysis is especially powerful when performed using the position weight matrices (PWM) for known transcription factors. E.g., using Homer [54], one can yield the list of exact regulators whose binding sites are overrepresented in the upstream regions of candidate genes. However, in metaRE we intentionally used a simpler consensus model for identification of overrepresented elements, making it more versatile and applicable for more organisms. First, for almost all organisms, including the model ones, the binding sites of most transcription factors remain unknown. Moreover, only very few organisms have PWMs for at least a hundred transcription factors (e.g., Saccharomyces cerevisiae, Arabidopsis thaliana, Drosophila melanogaster, Caenorhabditis elegans, Mus musculus, Homo sapiens) [56]. Second, metaRE could be applied not only to the upstream regions but to any sequences associated with the genes to find the signals unrelated to transcription factor DNA binding and not described by PWMs. For example, analyzing the 3'UTR metaRE could help identify the sites for the miRNA seeds binding. Third, in the present study of cold-responsive cis-elements, consensus search in metaRE with the subsequent analysis of identified sequences using PWMs for known transcription factors in TOMTOM [24] was shown to be very fruitful, with more than $65 \%$ of the elements annotated in Arabidopsis. We believe that the hybrid approaches with preliminary screening for enriched consensuses and subsequent annotation and reanalysis of the data using more powerful models are in need. Like an approach used in the study to annotate transcription factor binding sites in Nannochloropsis spp. microalgae [57].

\subsection{Hypothermia-Related and Cold-Stress Responsive Cistromes in Zebrafish and Arabidopsis}

Here, we employ metaRE in the investigation of widely studied processes, cold stress response, in which molecular mechanisms are still full of gaps. We performed an analysis using datasets generated for model objects in plant and animal fields, Arabidopsis, and zebrafish.

For plants, the cold stress response was studied in more detail, so that we were able to infer more data. Large-scale transcriptome studies showed that the CBF1-3, the major regulators of cold acclimation, in fact, regulate only a small portion of cold-responsive genes $[27,30,45,58]$ which means that other regulators may exist. Here, we see that CBFs binding sites are, indeed, not overrepresented in early cold stress response as the potential binding sites for other transcription factors (Table S2). CBFs binding sites seemed to be the most overrepresented in the late response (Table S3), which explains why only a small portion of cold-responsive genes are CBF-regulated. The most significantly enriched cis-element in early cold stress response detected by metaRE was the potential binding site for CAMTA (Figure 1, Table S1). CAMTA1-3 are known upstream regulators of CBF1-3, they increase freezing tolerance via activation of $\sim 15 \%$ cold-responsive genes $[28,40]$.

Park et al. (2015) found that, in parallel with CBF genes, 27 other "first-wave" transcription factor genes were highly upregulated at an early stage of cold treatment. Analysis of gene expression in transgenic plants overexpressing 11 of these first-wave transcription factors identified four transcription factors from bZIP family (ZAT12, ZF, ZAT10, and CZF1) and heat-shock factor HSFC1 involved in the regulation of cold-stress-responsive genes $[27,45,59]$. metaRE identified bZIP transcription factors binding sites as one of the most significantly enriched in promoters of early responsive to cold genes (Figure 2; Table S2), however, their impact was not that big in the late response.

Another interesting result relates to the cis-elements overrepresented in the promoters of downregulated by cold genes, which regulatory mechanisms are completely unknown. Here, we found potential binding sites for GATA and TCP transcription factors, as well as many unknown motifs.

A further experimental study is required to clarify the role of predicted unknown cis-elements, they could be rare versions of transcription factors binding sites, or form the biochemical environment for transcription factors binding, or be involved in chromatin structure formation. Anyway, to study these hypotheses experimental investigations are required. The role of candidate genes like GATA, HD-Zip, TCP, and others in the cold stress response still lacks the total understanding and explanation which we need to search for with experimental approaches. 
Although we found a great number of transcriptomes generated on zebrafish under suboptimal temperatures, the mechanisms of cold acclimation for this animal appeared to be largely unknown. In one of the few studies, a comprehensive analysis of the cis-regulatory code behind the low-temperature response has been performed [33]. In 16 RNA-Seq experiments, the authors inferred 33 gene clusters with common or tissue-specific expression patterns and then searched with the DREME tool [60] for cis-elements overrepresented in the clusters. As a result, they identified 17 octamers, overrepresented in one of the clusters, and experimentally verified two of them, AG(A/C)AACCA and (C/G)AGTCA. Here, we have applied an alternative strategy to search for the systematically enriched cis-elements over the same set of transcriptomes using metaRE. Notably, but not unexpectedly, that the cis-elements identified by [33] and in the present study were largely different; however, we both detected Nfya binding sites and a set of A/T-rich elements.

An exciting finding was that cis-elements detected in two separate metaRE studies for Arabidopsis and zebrafish significantly overlap by 27 hexamers. E-box motif CACGTG was highly overrepresented in promoters of both hypothermia-induced zebrafish' and cold-stress-induced Arabidopsis' genes. The E-box elements are known to be bound by bHLH transcription factors in many species including Arabidopsis and zebrafish [61]. In zebrafish, bHLH are involved in the control of developmental processes, one of which muscle development-is highly influenced by cold exposure [62]. The experimental study of E-box in the promoter of circadian clock gene Per4 showed that the amplitude of E-box-driven rhythmic expression response to temperature [52].

In both searches, metaRE detected the overrepresentation of A/T-rich sequences. Earlier, we got a similar result for auxin-regulated cistrome in Arabidopsis [9], but not in other studies (data not shown). The role of A/T-rich sequences can be different: they might be the parts of A/T-rich transcription factors binding sites (e.g., for Homeobox Factors), or they might be the TATA-box sequences, or they might be a part of chromatin landscape. The half of A/T-rich sequences identified for Arabidopsis were annotated by TOMTOM either as HD-ZIP binding sites or as TATA-boxes. As for Danio rerio, A/T-rich motifs were recognized as the potential binding sites of ZF (Zinc Finger) and Homeobox transcription factors. Homeobox transcription factors are known as development regulators [63]. Since exposure to low-temperatures crucially influences the developmental processes their involvement could be required. Unannotated AT-rich sequences still can predict a specific epigenetic landscape; in plants, cold-induced genes show enhanced chromatin accessibility, and a large number of active genes in cold-stored potato tubers are associated with a bivalent H3K4me3-H3K27me3 mark [64].

Temperature response is one of the basic stress responses with which primitive organisms had to cope millions of years before the separation of plant and animal kingdoms in evolution. Thus, we believe that comparative studies of the cis-elements conservation between plants and animals will help to clarify the mechanisms of low-temperature response. To do that, a more rigorous meta-analysis study on many organisms is in need. metaRE provides a framework of how this can be studied when a sufficient number of transcriptomes is generated.

Supplementary Materials: The following are available online at http://www.mdpi.com/2073-4425/11/6/634/s1, Figure S1: The percentage of A/T-rich motifs among predicted $k$-mers $(n=6-8)$ detected in promoters of differentially expressed genes with different settings ( $\mathrm{nFC}$ - no threshold for fold-change; FC1.5 - the threshold is fold-change 1.5; FC2 is fold-change 2)., Table S1: The list of cold-stress-responsive transcriptome datasets taken for meta-analysis with metaRE, Table S2: Cis-elements associated with early cold stress response on Arabidopsis, Table S3: Cis-elements associated with late cold stress response on Arabidopsis, Table S4: Cis-elements associated with hypothermia on zebrafish.

Author Contributions: Methodology, V.V.M., D.D.N., and P.A.C.; software, P.A.C.; validation and investigation, V.V.M., D.D.N., P.A.C., and Y.G.S.; writing—original draft preparation, D.D.N. and V.V.M.; writing-review and editing, V.V.M., D.D.N., P.A.C., and Y.G.S.; visualization, D.D.N., P.A.C., and Y.G.S.; supervision, V.V.M.; funding acquisition, V.V.M. All authors have read and agreed to the published version of the manuscript.

Funding: metaRE development was funded by Russian Foundation for Basic Research, grant number 18-04-01130, and by Russian State Budget, project number 0259-2019-0008-C-01. D.D.N. was supported by a PhD sandwich fellowship from Wageningen Graduate School. The study of cold stress response was supported by Russian Science Foundation, grant number 18-74-10008. 
Acknowledgments: We thank Ivo Grosse, Nadya Omelyanchuk, Jian Xu and Dolf Weijers for fruitful discussions and inspiration. We are grateful to Viktor Levitsky for consultations on the technical part of the algorithm. We acknowledge the Center of Shared Facilities "Bioinformatics" in IC\&G for providing us an access to high performance computing facilities.

Conflicts of Interest: The authors declare no conflicts of interest. The funders had no role in the design of the study; in the collection, analyses, or interpretation of data; in the writing of the manuscript, or in the decision to publish the results.

\section{References}

1. Rhodes, D.R.; Yu, J.; Shanker, K.; Deshpande, N.; Varambally, R.; Ghosh, D.; Barrette, T.; Pandey, A.; Chinnaiyan, A.M. Large-scale meta-analysis of cancer microarray data identifies common transcriptional profiles of neoplastic transformation and progression. Proc. Natl. Acad. Sci. USA 2004, 101, 9309-9314. [CrossRef] [PubMed]

2. Phan, J.H.; Young, A.N.; Wang, M.D. Robust Microarray Meta-Analysis Identifies Differentially Expressed Genes for Clinical Prediction. Sci. World J. 2012, 2012, 1-9. [CrossRef] [PubMed]

3. Shafi, A.; Nguyen, T.; Peyvandipour, A.; Draghici, S. GSMA: An approach to identify robust global and test Gene Signatures using Meta-Analysis. Bioinformatics 2019, btz561. [CrossRef] [PubMed]

4. Bargmann, B.O.R.; Vanneste, S.; Krouk, G.; Nawy, T.; Efroni, I.; Shani, E.; Choe, G.; Friml, J.; Bergmann, D.C.; Estelle, M.; et al. A map of cell type-specific auxin responses. Mol. Syst. Biol. 2013, 9, 688. [CrossRef]

5. Vogel, J.T.; Zarka, D.G.; Van Buskirk, H.A.; Fowler, S.G.; Thomashow, M.F. Roles of the CBF2 and ZAT12 transcription factors in configuring the low temperature transcriptome of Arabidopsis: Arabidopsis low temperature transcriptome. Plant J. 2004, 41, 195-211. [CrossRef]

6. He, H.; Cao, S.; Niu, T.; Zhou, Y.; Zhang, L.; Zeng, Y.; Zhu, W.; Wang, Y.; Deng, H. Network-Based Meta-Analyses of Associations of Multiple Gene Expression Profiles with Bone Mineral Density Variations in Women. PLoS ONE 2016, 11, e0147475. [CrossRef]

7. Ament, S.A.; Blatti, C.A.; Alaux, C.; Wheeler, M.M.; Toth, A.L.; Le Conte, Y.; Hunt, G.J.; Guzman-Novoa, E.; DeGrandi-Hoffman, G.; Uribe-Rubio, J.L.; et al. New meta-analysis tools reveal common transcriptional regulatory basis for multiple determinants of behavior. Proc. Nat. Acad. Sci. USA 2012, 109, E1801-E1810. [CrossRef]

8. Schwarz, B.; Azodi, C.B.; Shiu, S.-H.; Bauer, P. Putative cis-Regulatory Elements Predict Iron Deficiency Responses in Arabidopsis Roots. Plant Physiol. 2020, 182, 1420-1439. [CrossRef]

9. Cherenkov, P.; Novikova, D.; Omelyanchuk, N.; Levitsky, V.; Grosse, I.; Weijers, D.; Mironova, V. Diversity of cis-regulatory elements associated with auxin response in Arabidopsis thaliana. J. Exp. Bot. 2018, 69, 329-339. [CrossRef]

10. Eddelbuettel, D. Seamless $R$ and $C++$ Integration with Rcpp; Springer: New York, NY, USA, 2013; ISBN 978-1-4614-6867-7.

11. Davis, S.; Meltzer, P.S. GEOquery: A bridge between the Gene Expression Omnibus (GEO) and BioConductor. Bioinformatics 2007, 23, 1846-1847. [CrossRef]

12. Ritchie, M.E.; Phipson, B.; Wu, D.; Hu, Y.; Law, C.W.; Shi, W.; Smyth, G.K. limma powers differential expression analyses for RNA-sequencing and microarray studies. Nucleic Acids Res. 2015, 43, 47. [CrossRef]

13. Robinson, M.D.; McCarthy, D.J.; Smyth, G.K. edgeR: A Bioconductor package for differential expression analysis of digital gene expression data. Bioinformatics 2010, 26, 139-140. [CrossRef]

14. McCarthy, D.J.; Chen, Y.; Smyth, G.K. Differential expression analysis of multifactor RNA-Seq experiments with respect to biological variation. Nucleic Acids Res. 2012, 40, 4288-4297. [CrossRef]

15. Edgar, R. Gene Expression Omnibus: NCBI gene expression and hybridization array data repository. Nucleic Acids Res. 2002, 30, 207-210. [CrossRef]

16. Pagès, H.; Aboyoun, P.; Gentleman, R.; DebRoy, S. Biostrings: Efficient Manipulation of Biological Strings. $R$ Package Version 2.56.0. 2020. Available online: https://bioconductor.org/packages/release/bioc/html/ Biostrings.html (accessed on 8 June 2020). [CrossRef]

17. Durinck, S.; Moreau, Y.; Kasprzyk, A.; Davis, S.; De Moor, B.; Brazma, A.; Huber, W. BioMart and Bioconductor: A powerful link between biological databases and microarray data analysis. Bioinformatics 2005, 21, 3439-3440. [CrossRef] 
18. Mironova, V.V.; Omelyanchuk, N.A.; Wiebe, D.S.; Levitsky, V.G. Computational analysis of auxin responsive elements in the Arabidopsis thaliana L. genome. BMC Genom. 2014, 15, S4. [CrossRef]

19. Zemlyanskaya, E.V.; Wiebe, D.S.; Omelyanchuk, N.A.; Levitsky, V.G.; Mironova, V.V. Meta-analysis of transcriptome data identified TGTCNN motif variants associated with the response to plant hormone auxin in Arabidopsis thaliana L. J. Bioinform. Comput. Biol. 2016, 14, 1641009. [CrossRef]

20. Holm, S. A simple sequentially rejective multiple test procedure. Scand. J. Stat. 1979, 6, 65-70.

21. Hochberg, Y. A sharper Bonferroni procedure for multiple tests of significance. Biometrika 1988, 75, 800-802. [CrossRef]

22. Benjamini, Y.; Hochberg, Y. Controlling the false discovery rate: A practical and powerful approach to multiple testing. J. R. Stat. Soc. Ser. B 1995, 57, 289-300. [CrossRef]

23. Benjamini, Y.; Yekutieli, D. The Control of the False Discovery Rate in Multiple Testing under Dependency. Ann. Stat. 2001, 29, 1165-1188.

24. Gupta, S.; Stamatoyannopoulos, J.A.; Bailey, T.L.; Noble, W. Quantifying similarity between motifs. Genome Biol. 2007, 8, R24. [CrossRef]

25. Guan, Q.; Wu, J.; Zhang, Y.; Jiang, C.; Liu, R.; Chai, C.; Zhu, J. A DEAD Box RNA Helicase Is Critical for Pre-mRNA Splicing, Cold-Responsive Gene Regulation, and Cold Tolerance in Arabidopsis. Plant Cell 2013, 25, 342-356. [CrossRef]

26. Chiba, Y.; Mineta, K.; Hirai, M.Y.; Suzuki, Y.; Kanaya, S.; Takahashi, H.; Onouchi, H.; Yamaguchi, J.; Naito, S. Changes in mRNA Stability Associated with Cold Stress in Arabidopsis Cells. Plant Cell Physiol. 2013, 54, 180-194. [CrossRef]

27. Park, S.; Lee, C.-M.; Doherty, C.J.; Gilmour, S.J.; Kim, Y.; Thomashow, M.F. Regulation of the Arabidopsis CBF regulon by a complex low-temperature regulatory network. Plant J. 2015, 82, 193-207. [CrossRef]

28. Kim, Y.; Park, S.; Gilmour, S.J.; Thomashow, M.F. Roles of CAMTA transcription factors and salicylic acid in configuring the low-temperature transcriptome and freezing tolerance of Arabidopsis. Plant J. 2013, 75, 364-376. [CrossRef]

29. Lee, B.; Henderson, D.A.; Zhu, J.-K. The Arabidopsis Cold-Responsive Transcriptome and Its Regulation by ICE1. Plant Cell 2005, 17, 3155-3175. [CrossRef]

30. Jia, Y.; Ding, Y.; Shi, Y.; Zhang, X.; Gong, Z.; Yang, S. The cbfs triple mutants reveal the essential functions of CBFs in cold acclimation and allow the definition of CBF regulons in Arabidopsis. New Phytol. 2016, 212, 345-353. [CrossRef]

31. Kilian, J.; Whitehead, D.; Horak, J.; Wanke, D.; Weinl, S.; Batistic, O.; D’Angelo, C.; Bornberg-Bauer, E.; Kudla, J.; Harter, K. The AtGenExpress global stress expression data set: Protocols, evaluation and model data analysis of UV-B light, drought and cold stress responses: AtGenExpress global abiotic stress data set. Plant J. 2007, 50, 347-363. [CrossRef]

32. Schlaen, R.G.; Mancini, E.; Sanchez, S.E.; Perez-Santángelo, S.; Rugnone, M.L.; Simpson, C.G.; Brown, J.W.S.; Zhang, X.; Chernomoretz, A.; Yanovsky, M.J. The spliceosome assembly factor GEMIN2 attenuates the effects of temperature on alternative splicing and circadian rhythms. Proc. Natl. Acad. Sci. USA 2015, 112, 9382-9387. [CrossRef] [PubMed]

33. Hu, P.; Liu, M.; Zhang, D.; Wang, J.; Niu, H.; Liu, Y.; Wu, Z.; Han, B.; Zhai, W.; Shen, Y.; et al. Global identification of the genetic networks and cis -regulatory elements of the cold response in zebrafish. Nucleic Acids Res. 2015, 43, 9198-9213. [CrossRef] [PubMed]

34. Durinck, S.; Spellman, P.T.; Birney, E.; Huber, W. Mapping identifiers for the integration of genomic datasets with the R/Bioconductor package biomaRt. Nat. Protoc. 2009, 4, 1184-1191. [CrossRef]

35. Thomashow, M.F. PLANT COLD ACCLIMATION: Freezing Tolerance Genes and Regulatory Mechanisms. Annu. Rev. Plant. Physiol. Plant. Mol. Biol. 1999, 50, 571-599. [CrossRef]

36. Gilmour, S.J.; Zarka, D.G.; Stockinger, E.J.; Salazar, M.P.; Houghton, J.M.; Thomashow, M.F. Low temperature regulation of theArabidopsisCBF family of AP2 transcriptional activators as an early step in cold-inducedCORgene expression. Plant J. 1998, 16, 433-442. [CrossRef]

37. Zarka, D.G.; Vogel, J.T.; Cook, D.; Thomashow, M.F. Cold Induction of Arabidopsis CBF Genes Involves Multiple ICE (Inducer of CBF Expression) Promoter Elements and a Cold-Regulatory Circuit That Is Desensitized by Low Temperature. Plant Physiol. 2003, 133, 910-918. [CrossRef] 
38. Novillo, F.; Alonso, J.M.; Ecker, J.R.; Salinas, J. CBF2/DREB1C is a negative regulator of CBF1/DREB1B and CBF3/DREB1A expression and plays a central role in stress tolerance in Arabidopsis. Proc. Natl. Acad. Sci. USA 2004, 101, 3985-3990. [CrossRef]

39. Yamaguchi-Shinozaki, K.; Shinozaki, K. Organization of cis-acting regulatory elements in osmotic- and cold-stress-responsive promoters. Trends Plant Sci. 2005, 10, 88-94. [CrossRef]

40. Doherty, C.J.; Van Buskirk, H.A.; Myers, S.J.; Thomashow, M.F. Roles for Arabidopsis CAMTA Transcription Factors in Cold-Regulated Gene Expression and Freezing Tolerance. Plant Cell 2009, 21, 972-984. [CrossRef]

41. Kidokoro, S.; Maruyama, K.; Nakashima, K.; Imura, Y.; Narusaka, Y.; Shinwari, Z.K.; Osakabe, Y.; Fujita, Y.; Mizoi, J.; Shinozaki, K.; et al. The Phytochrome-Interacting Factor PIF7 Negatively Regulates DREB1 Expression under Circadian Control in Arabidopsis. Plant Physiol. 2009, 151, 2046-2057. [CrossRef]

42. Lee, C.-M.; Thomashow, M.F. Photoperiodic regulation of the C-repeat binding factor (CBF) cold acclimation pathway and freezing tolerance in Arabidopsis thaliana. Proc. Natl. Acad. Sci. USA 2012, 109, 15054-15059. [CrossRef]

43. Maruyama, K.; Todaka, D.; Mizoi, J.; Yoshida, T.; Kidokoro, S.; Matsukura, S.; Takasaki, H.; Sakurai, T.; Yamamoto, Y.Y.; Yoshiwara, K.; et al. Identification of Cis-Acting Promoter Elements in Cold- and Dehydration-Induced Transcriptional Pathways in Arabidopsis, Rice, and Soybean. DNA Res. 2012, 19, 37-49. [CrossRef] [PubMed]

44. Jiang, B.; Shi, Y.; Zhang, X.; Xin, X.; Qi, L.; Guo, H.; Li, J.; Yang, S. PIF3 is a negative regulator of the CBF pathway and freezing tolerance in Arabidopsis. Proc. Natl. Acad. Sci. USA 2017, 114, E6695-E6702. [CrossRef]

45. Zhao, C.; Zhang, Z.; Xie, S.; Si, T.; Li, Y.; Zhu, J.-K. Mutational Evidence for the Critical Role of CBF Genes in Cold Acclimation in Arabidopsis. Plant Physiol. 2016, 533. [CrossRef]

46. Agarwal, M.; Hao, Y.; Kapoor, A.; Dong, C.-H.; Fujii, H.; Zheng, X.; Zhu, J.-K. A R2R3 Type MYB Transcription Factor Is Involved in the Cold Regulation of CBF Genes and in Acquired Freezing Tolerance. J. Biol. Chem. 2006, 281, 37636-37645. [CrossRef]

47. Dong, M.A.; Farre, E.M.; Thomashow, M.F. CIRCADIAN CLOCK-ASSOCIATED 1 and LATE ELONGATED HYPOCOTYL regulate expression of the C-REPEAT BINDING FACTOR (CBF) pathway in Arabidopsis. Proc. Natl. Acad. Sci. USA 2011, 108, 7241-7246. [CrossRef]

48. Kidokoro, S.; Yoneda, K.; Takasaki, H.; Takahashi, F.; Shinozaki, K.; Yamaguchi-Shinozaki, K. Different Cold-Signaling Pathways Function in the Responses to Rapid and Gradual Decreases in Temperature. Plant Cell 2017, 29, 760-774. [CrossRef]

49. Yang, S.-H.; Sharrocks, A.D. Convergence of the SUMO and MAPK pathways on the ETS-domain transcription factor Elk-1. Biochem. Soc. Symp. 2006, 73, 121-129. [CrossRef]

50. Ducker, C.; Chow, L.K.Y.; Saxton, J.; Handwerger, J.; McGregor, A.; Strahl, T.; Layfield, R.; Shaw, P.E. De-ubiquitination of ELK-1 by USP17 potentiates mitogenic gene expression and cell proliferation. Nucleic Acids Res. 2019, 47, 4495-4508. [CrossRef]

51. Felber, K.; Elks, P.M.; Lecca, M.; Roehl, H.H. Expression of osterix Is Regulated by FGF and Wnt/ $\beta$-Catenin Signalling during Osteoblast Differentiation. PLoS ONE 2015, 10, e0144982. [CrossRef]

52. Lahiri, K.; Vallone, D.; Gondi, S.B.; Santoriello, C.; Dickmeis, T.; Foulkes, N.S. Temperature Regulates Transcription in the Zebrafish Circadian Clock. PLoS Biol. 2005, 3, e351. [CrossRef]

53. van Helden, J.; André, B.; Collado-Vides, J. A web site for the computational analysis of yeast regulatory sequences. Yeast 2000,16,177-187. [CrossRef]

54. Heinz, S.; Benner, C.; Spann, N.; Bertolino, E.; Lin, Y.C.; Laslo, P.; Cheng, J.X.; Murre, C.; Singh, H.; Glass, C.K. Simple Combinations of Lineage-Determining Transcription Factors Prime cis-Regulatory Elements Required for Macrophage and B Cell Identities. Mol. Cell 2010, 38, 576-589. [CrossRef] [PubMed]

55. Liu, T.; Ortiz, J.A.; Taing, L.; Meyer, C.A.; Lee, B.; Zhang, Y.; Shin, H.; Wong, S.S.; Ma, J.; Lei, Y.; et al. Cistrome: An integrative platform for transcriptional regulation studies. Genome Biol. 2011, 12, R83. [CrossRef]

56. Khan, A.; Fornes, O.; Stigliani, A.; Gheorghe, M.; Castro-Mondragon, J.A.; van der Lee, R.; Bessy, A.; Chèneby, J.; Kulkarni, S.R.; Tan, G.; et al. JASPAR 2018: Update of the open-access database of transcription factor binding profiles and its web framework. Nucleic Acids Res. 2018, 46, D260-D266. [CrossRef] [PubMed]

57. Hu, J.; Wang, D.; Li, J.; Jing, G.; Ning, K.; Xu, J. Genome-wide identification of transcription factors and transcription-factor binding sites in oleaginous microalgae Nannochloropsis. Sci. Rep. 2015, 4, 5454. [CrossRef] 
58. Chinnusamy, V.; Zhu, J.; Zhu, J.-K. Cold stress regulation of gene expression in plants. Trends Plant Sci. 2007, 12, 444-451. [CrossRef]

59. Zhao, C.; Lang, Z.; Zhu, J.-K. Cold responsive gene transcription becomes more complex. Trends Plant Sci. 2015, 20, 466-468. [CrossRef] [PubMed]

60. Bailey, T.L. DREME: Motif discovery in transcription factor ChIP-seq data. Bioinformatics 2011, 27, 1653-1659. [CrossRef]

61. Chen, Y.-H.; Lee, W.-C.; Cheng, C.-H.; Tsai, H.-J. Muscle regulatory factor gene: Zebrafish (Danio rerio) myogenin cDNA. Comp. Biochem. Physiol. Part B Biochem. Mol. Biol. 2000, 127, 97-103. [CrossRef]

62. Campos, C.; Valente, L.; Conceição, L.; Engrola, S.; Fernandes, J. Temperature affects methylation of the myogenin putative promoter, its expression and muscle cellularity in Senegalese sole larvae. Epigenetics 2013, 8, 389-397. [CrossRef] [PubMed]

63. Goulding, M.D.; Gruss, P. The homeobox in vertebrate development. Curr. Opin. Cell Biol. 1989, 1, $1088-1093$. [CrossRef]

64. Zeng, Z.; Zhang, W.; Marand, A.P.; Zhu, B.; Buell, C.R.; Jiang, J. Cold stress induces enhanced chromatin accessibility and bivalent histone modifications H3K4me3 and H3K27me3 of active genes in potato. Genome Biol. 2019, 20, 123. [CrossRef] [PubMed]

(C) 2020 by the authors. Licensee MDPI, Basel, Switzerland. This article is an open access article distributed under the terms and conditions of the Creative Commons Attribution (CC BY) license (http://creativecommons.org/licenses/by/4.0/). 